\title{
Diabetes Management via Mobile Phones: A Systematic Review
}

\author{
Bree Holtz, Ph.D., ${ }^{1}$ and Carolyn Lauckner, B.A. ${ }^{2}$ \\ ${ }^{1}$ Center for Clinical Management Research, Veteran Affairs Ann \\ Arbor Health Care System, Ann Arbor, Michigan. \\ ${ }^{2}$ Telecommunications, Information Studies \& Media, Michigan \\ State University, East Lansing, Michigan.
}

\begin{abstract}
Background: This study sought to understand the most common uses and functions of mobile phones in monitoring and managing diabetes, their potential role in a clinical setting, and the current state of research in this area. Methods: We identified peer-reviewed articles published between 2000 and 2010. Twenty-one articles were analyzed for this systematic literature review. Results: The majority of studies examined the use of mobile phones from the patient's perspective. Subjects with type 1 diabetes were enrolled exclusively in over 50\% of the studies. Seventy-one percent of the studies used a study-specific application, which had supplemental features in addition to text messaging. The outcomes assessed varied considerably across studies, but some positive trends were noted, such as improved self-efficacy, hemoglobin A1c, and self-management behaviors. Conclusions: The studies evaluated showed promise in using mobile phones to help people with diabetes manage their condition effectively. However, many of these studies lacked sufficient sample sizes or intervention lengths to determine whether the results might be clinically or statistically significant. Future research should examine other key issues, such as provider perceptions, integration into a healthcare practice, and cost, which would provide important insight into the use of mobile phones for chronic disease management.
\end{abstract}

Key words: home health monitoring, telecommunications, telehealth

\section{Introduction}

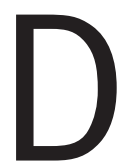

iabetes is a well-documented health problem in the United States and worldwide. The Centers for Disease Control and Prevention reports that about 25.8 million people (8.3\%) in the United States have been diagnosed with this disease. ${ }^{1}$ Worldwide, approximately 220 million people have diabetes, and estimates suggest this number will grow to 366 million by $2030 .^{2}$ Past research has found that patients who use self-monitoring techniques, including monitoring food intake, physical activity, and glucose levels, have better control of their disease. ${ }^{3,4}$

One way that has been proposed to help individuals better manage their diabetes is through use of mobile phones, which are now widely available, offer a variety of communication methods, and are rela- tively inexpensive. The availability of mobile phones worldwide is growing, and at the end of 2010 the International Telecommunications Union posits that there will be an estimated 5.3 billion mobile cellular subscriptions. ${ }^{5}$ Text messaging, also known as short message service (SMS), is a relatively low cost way to send asynchronous messages via mobile phones and is increasing in popularity. An estimated 200,000 text messages are sent every second. ${ }^{5}$ In the United States, among teenagers (12-17 years old) who have a mobile phone, over 54\% send daily text messages, and almost half send 50 or more text messages per day. ${ }^{6}$ Additionally, a recent report from the Pew Research Center ${ }^{7}$ states that $7 \%$ of mobile phone users have used their phone to search for health or medical information. This trend is higher among younger people, as 29\% of 18-29 year olds have performed this type of search. The use and prevalence of mobile applications are also on the rise, as evidenced by the fact that a search for "Diabetes" within the iPhone ${ }^{\circledR}$ (Apple) App store yields 262 results as of March 2011.

Because of the seemingly ubiquitous nature of mobile phones, many researchers and health providers have used mobile phones as a way to educate or help people to manage their health issues. ${ }^{8-12}$ Similar to previous research that has examined the use of mobile phones for diabetes, ${ }^{10}$ this study sought to examine the types of mobile phone-based interventions implemented among people with diabetes and the potential effect of these interventions on patient outcomes.

\section{Methods \\ SEARCH STRATEGY}

This study began by searching the following electronic databases: Science Citation Index Expanded, Social Sciences Index, Art \& Humanities Citation Index, and ProQuest for peer-reviewed articles published between 2000 and May 2010. Search terms included various combinations of the terms "diabetes," "diabetes mellitus," "mobile phone," "cell phone," "cellular phone," "text messaging," "text message," "SMS," and "short message service." References of identified articles were also searched for potential articles for inclusion. Only articles published in peer-reviewed scientific journals in English were eligible for review. The studies reviewed also had to use the mobile phone as the primary device of the intervention.

\section{STUDY SELECTION}

We identified 28 articles that met the basic criteria of our search. However, when the articles were further reviewed, seven articles were excluded, primarily because the mobile phone was not the main study intervention technology or the article was a description of a planned intervention that had not yet been implemented. Thus, in 


\section{HOLTZ AND LAUCKNER}

total, 21 articles were analyzed for this systematic literature review (Table 1). All articles were independently reviewed and coded by the authors, and the following data were extracted: self-care/ management activities (i.e., glucose monitoring, eating/diet, physical activity), method of intervention (i.e., application, text messaging only, combination), intervention activity (i.e., diary/log, reminder, informational), outcomes measured (i.e., hemoglobin A1c [HbA1c], body mass index [BMI], self-efficacy, knowledge, satisfaction/ usefulness), and method of data transmittal. Additionally, study design and duration, type and glycemic control of diabetes, sample size, study participant (i.e., patient, physician, nurse, informal caregiver) recruitment process, phone ownership, location, costs, and reported technical issues were examined (Table 2). Both coders had previous coding experience and were well versed in the area of mobile health. During the process of establishing reliability, the coding scheme was refined and explicated as necessary. Once reliability was established (Krippendorff's alpha $\geq 0.8$ for each coded item), any discrepancies between the two reviewers were subject to multiple reviews and then settled by consensus.

\section{Results}

\section{STUDY DESIGNS AND SUBJECTS}

The majority (95\%) of studies examined the use of mobile phones from the patient perspective, while 19\% took into account the healthcare providers (physicians [14\%], nurses [5\%]), and two studies (9.5\%) included informal caregivers, such as parents of the patient. Sample sizes ranged from 6 to 100 subjects, with a mean of 38 subjects and a median number of 30. Subjects with type 1 diabetes were used exclusively in 57\% of the studies, 19\% had patients with type 2 diabetes only, $10 \%$ of the studies used patients with both types, and $14 \%$ of the studies did not report this information. Level of glycemic control, as measured by HbA1c, was reported in 57\% of studies.

Almost half of the studies had some type of participant randomization (47.6\%). Two studies utilized a crossover design in their intervention. The length of the interventions varied from 2 weeks to 1 year. The average length of intervention was 22.5 weeks, excluding two studies that did not report intervention length. Fourteen of the 21 studies described participant inclusion and exclusion criteria. Having a comorbidity was the most commonly stated exclusionary factor (23.8\%), whereas the most commonly reported inclusion criterion involved participant age (62\%). Treatment with insulin was required by one-third of studies. Seventy-one percent of studies explicitly stated the location of the intervention; these studies took place in the United Kingdom $(n=5)$, the United States $(n=4)$, Scandinavia $(n=3)$, and continental Europe $(n=2)$.

\section{MOBILE PHONE TECHNOLOGIES}

Over half of studies (57\%) provided a mobile phone to the subjects, whereas 14\% had subjects use their own phone, and 29\% did not report this information. One-third of studies stated that the study paid for the mobile phone service, and two-thirds of studies did not report who paid for the service. Additionally, four studies discussed overall cost data. Technical issues, such as lost messages or limitations of coverage area, were reported in $67 \%$ of studies.

Seventy-one percent of studies used a study-specific application, which is a program that was developed for the study and has more functionalities or features than simple text messaging. The type of function (i.e., diary/log, reminders, information/education) used in the intervention varied, and many studies included multiple functions. Messages that reminded the participant to do an activity were used in 52\% of the studies. Six studies (39\%) used the messages as an opportunity to educate the participants with tips and information about diabetes. Eighty-one percent of studies reported using a diary function to record data such as blood glucose readings, carbohydrate or calorie consumption, or physical activity. The most common transmission methods of blood glucose values were Bluetooth ${ }^{\circledR}$ (Bluetooth SIG, Inc.) (62.5\%), a physical wire to the phone (12.5\%), or infrared signaling $(12.5 \%)$ between the phone and the glucometer. Studies that did not develop or use a study-specific application used text messaging only. In these instances, subjects had to manually enter their information into the mobile phone and send it.

\section{OUTCOMES REPORTED}

The studies reviewed used many different outcome measures, making it difficult to do a rigorous analysis of the clinical findings. Outcomes related to self-care and management activities were reported by $43 \%$ of studies. These activities included glucose monitoring (67\%), eating (44\%), and exercise (44\%). Sixty-two percent of studies reported $\mathrm{HbA1c}$ as an outcome measure, of which $85 \%$ reported improvements. However, statistically significant changes in $\mathrm{HbA1c}$ were reported in only three of the studies. BMI was an outcome in $24 \%$ of studies, and no significant changes were found. Selfefficacy was reported as an outcome by $24 \%$ of studies, and all found nonsignificant improvements. Knowledge about diabetes and diabetes management was reported as an outcome in 14.3\% of studies, with improvements reported in two studies. Information about the costs associated with this type of intervention was reported in four studies. Of the $48 \%$ of studies that reported satisfaction as an outcome measure, 90\% of them reported that the subjects were satisfied with this type of intervention.

\section{Discussion}

This review highlights the work that has been done in using mobile phones to help people with diabetes manage their disease and improve health and behavior outcomes. When one considers the ubiquity of mobile phones in modern life and their increasing use for health applications, the amount of research conducted about using them for diabetes management seems comparatively small. The studies evaluated demonstrate many positive trends, but few significant findings were reported. The small number of significant findings could be due to the small sample sizes; the average number of subjects in the studies reviewed here was 38, and just under half were randomized to a condition. Also worth noting is the fact that many of the studies did not report power calculations. Thus, overall, the generalizability of the data as they relate to other populations is 


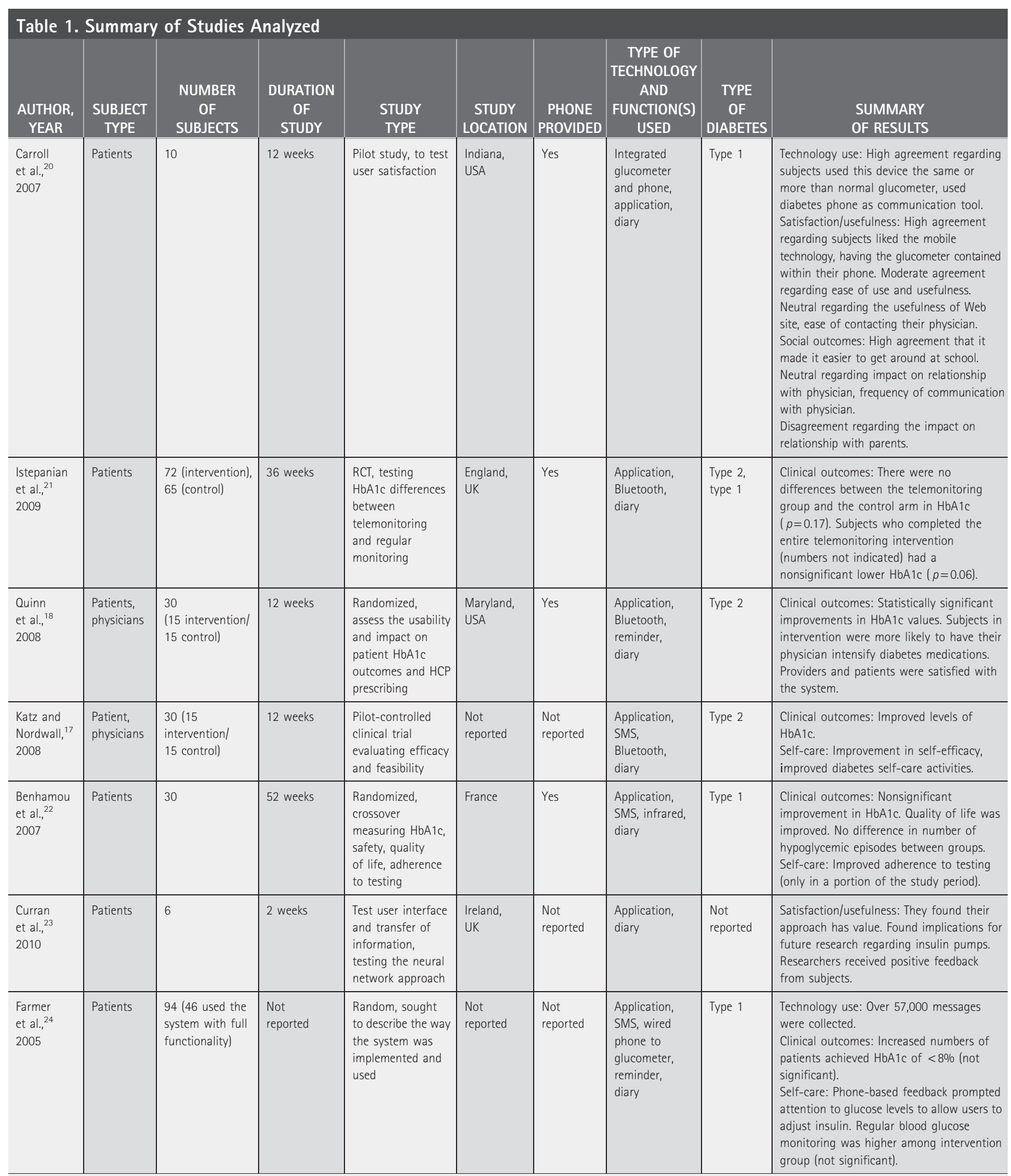




\section{HOLTZ AND LAUCKNER}

\begin{tabular}{|c|c|c|c|c|c|c|c|c|c|}
\hline $\begin{array}{l}\text { AUTHOR, } \\
\text { YEAR }\end{array}$ & $\begin{array}{l}\text { SUBJECT } \\
\text { TYPE }\end{array}$ & $\begin{array}{l}\text { NUMBER } \\
\text { OF } \\
\text { SUBJECTS }\end{array}$ & $\begin{array}{c}\text { DURATION } \\
\text { OF } \\
\text { STUDY }\end{array}$ & $\begin{array}{l}\text { STUDY } \\
\text { TYPE }\end{array}$ & $\begin{array}{c}\text { STUDY } \\
\text { LOCATION }\end{array}$ & $\begin{array}{c}\text { PHONE } \\
\text { PROVIDED }\end{array}$ & $\begin{array}{c}\text { TYPE OF } \\
\text { TECHNOLOGY } \\
\text { AND } \\
\text { FUNCTION(S) } \\
\text { USED }\end{array}$ & $\begin{array}{c}\text { TYPE } \\
\text { OF } \\
\text { DIABETES }\end{array}$ & $\begin{array}{l}\text { SUMMARY } \\
\text { OF RESULTS }\end{array}$ \\
\hline $\begin{array}{l}\text { Faridi } \\
\text { et al.,., } \\
2008\end{array}$ & Patients & $\begin{array}{l}30 \text { (15 } \\
\text { intervention/ } \\
15 \text { control) }\end{array}$ & 12 weeks & $\begin{array}{l}\text { Random, test } \\
\text { feasibility, clinical } \\
\text { outcomes (HbA1c, } \\
\text { BMI, blood } \\
\text { pressure, physical } \\
\text { activity, self-care, } \\
\text { and self-efficacy) }\end{array}$ & $\begin{array}{l}\text { Connecticut, } \\
\text { USA }\end{array}$ & $\begin{array}{l}\text { Not } \\
\text { reported }\end{array}$ & $\begin{array}{l}\text { SMS, wireless } \\
\text { biometric } \\
\text { devices to } \\
\text { transmit } \\
\text { clinical data } \\
\text { that transmits } \\
\text { tailored } \\
\text { feedback } \\
\text { to patient } \\
\text { via SMS, } \\
\text { reminder }\end{array}$ & Type 2 & $\begin{array}{l}\text { Technology use: Two subjects in the } \\
\text { intervention group were completely } \\
\text { adherent; four subjects used the } \\
\text { intervention for } 1-2 \text { months; four subjects } \\
\text { used it for } 1 \text { week; five did not transmit } \\
\text { information. } \\
\text { Satisfaction/usefulness: Technical problems } \\
\text { were reported with equipment. Usability } \\
\text { issues: Too many menus, small buttons, } \\
\text { commands changed frequently. Most } \\
\text { preferred usual diabetic self-management. }\end{array}$ \\
\hline $\begin{array}{l}\text { Ferrer-Roca } \\
\text { et al.. }{ }^{25} \\
2004\end{array}$ & Patients & 23 & 32 weeks & $\begin{array}{l}\text { User satisfaction, } \\
\text { system use, } \\
\text { and cost data } \\
\text { were analyzed. }\end{array}$ & $\begin{array}{l}\text { Not } \\
\text { reported }\end{array}$ & $\begin{array}{l}\text { Subjects } \\
\text { used own } \\
\text { phone }\end{array}$ & SMS, diary & $\begin{array}{l}\text { Not } \\
\text { reported }\end{array}$ & $\begin{array}{l}\text { Technology use: Average of } 33 \text { SMS } \\
\text { messages per month from } 23 \text { subjects. } \\
\text { Reduction of messages during the holidays. } \\
\text { Satisfaction/usefulness: Overall user } \\
\text { satisfaction was good ( } n=6 \text { responses). } \\
\text { Cost: The cost analysis was based on } \\
\text { subjects using their own phone and SIM } \\
\text { card and estimate } € 3 / \text { month for the user } \\
\text { and the cost of the manager to be } € 3.75 / \\
\text { month per patient. }\end{array}$ \\
\hline $\begin{array}{l}\text { Franklin } \\
\text { et al., } \\
2008\end{array}$ & Patients & 64 & 52 weeks & $\begin{array}{l}\text { Utility of } \\
\text { intervention } \\
\text { and examine } \\
\text { user interaction }\end{array}$ & $\begin{array}{l}\text { Not } \\
\text { reported }\end{array}$ & Yes & $\begin{array}{l}\text { SMS, reminder, } \\
\text { entertainment, } \\
\text { information/ } \\
\text { educational } \\
\text { message }\end{array}$ & Type 1 & $\begin{array}{l}\text { Technology use: 1,180 messages were } \\
\text { submitted during the study period. Five } \\
\text { subjects used the system the majority of } \\
\text { the time (52\% of all messages). Female } \\
\text { subjects sent more messages not regarding } \\
\text { diabetes. Unprompted submission of blood } \\
\text { glucose was most common message type. } \\
\text { Responses to requests for personal } \\
\text { experience and tips accounted for } 40 \% \\
\text { of incoming messages as well as asking } \\
\text { questions and ordering supplies. Subjects } \\
\text { did not request that an outside (of the } \\
\text { study) person receive texts regarding the } \\
\text { subjects' status. }\end{array}$ \\
\hline $\begin{array}{l}\text { Gammon } \\
\text { et al..1 } \\
2005\end{array}$ & $\begin{array}{l}\text { Patients } \\
\text { and } \\
\text { informal } \\
\text { caregivers }\end{array}$ & $\begin{array}{l}30 \text { ( } 15 \text { children/ } \\
15 \text { parents) }\end{array}$ & 16 weeks & $\begin{array}{l}\text { Self-selected } \\
\text { child (with } \\
\text { diabetes)-parent } \\
\text { dyads were used, } \\
\text { both user groups } \\
\text { were asked } \\
\text { about use } \\
\text { and satisfaction, } \\
\text { and some parents } \\
\text { were interviewed } \\
\text { regarding } \\
\text { experiences, } \\
\text { advantages and } \\
\text { disadvantages. }\end{array}$ & Norway & $\begin{array}{l}\text { Yes (for } \\
\text { children/ } \\
\text { parents } \\
\text { used own } \\
\text { phones) }\end{array}$ & $\begin{array}{l}\text { Application, } \\
\text { SMS, } \\
\text { Bluetooth, } \\
\text { diary }\end{array}$ & Type 1 & $\begin{array}{l}\text { Technology use: System was used 3-4 } \\
\text { times/day. } \\
\text { Satisfaction/usefulness: When the children } \\
\text { were away from home, both groups } \\
\text { thought the automatic transfer of blood } \\
\text { glucose levels was good. The parents had } \\
\text { higher levels of satisfaction than the } \\
\text { children. All parents felt the system } \\
\text { provided reassurance. Parents liked } \\
\text { knowing if their child has measured his or } \\
\text { her blood glucose. Information provided } \\
\text { was good for recently diagnosed children. } \\
\text { Individuals thought the system should be } \\
\text { more automated. } \\
\text { Self-care: Living with diabetes was easier } \\
\text { with the phone. } \\
\text { Social outcomes: Mixed interview findings } \\
\text { regarding nagging, independence of } \\
\text { self-management, and surveillance } \\
\text { of self-management activities. }\end{array}$ \\
\hline
\end{tabular}




\begin{tabular}{|c|c|c|c|c|c|c|c|c|c|}
\hline $\begin{array}{l}\text { AUTHOR, } \\
\text { YEAR }\end{array}$ & $\begin{array}{l}\text { SUBJECT } \\
\text { TYPE }\end{array}$ & $\begin{array}{l}\text { NUMBER } \\
\text { OF } \\
\text { SUBJECTS }\end{array}$ & $\begin{array}{c}\text { DURATION } \\
\text { OF } \\
\text { STUDY }\end{array}$ & $\begin{array}{l}\text { STUDY } \\
\text { TYPE }\end{array}$ & $\begin{array}{c}\text { STUDY } \\
\text { LOCATION }\end{array}$ & $\begin{array}{c}\text { PHONE } \\
\text { PROVIDED }\end{array}$ & $\begin{array}{c}\text { TYPE OF } \\
\text { TECHNOLOGY } \\
\text { AND } \\
\text { FUNCTION(S) } \\
\text { USED }\end{array}$ & \begin{tabular}{|c|} 
TYPE \\
OF \\
DIABETES
\end{tabular} & $\begin{array}{l}\text { SUMMARY } \\
\text { OF RESULTS }\end{array}$ \\
\hline $\begin{array}{l}\text { Vähätalo } \\
\text { et al.,14 } \\
2004\end{array}$ & Patients & $\begin{array}{l}203(101 \\
\text { control/102 } \\
\text { intervention) }\end{array}$ & 52 weeks & $\begin{array}{l}\text { Use of the } \\
\text { application, } \\
\text { weight, insulin } \\
\text { dose, and HbA1c }\end{array}$ & Finland & Yes & $\begin{array}{l}\text { Application, } \\
\text { SMS, diary, } \\
\text { information }\end{array}$ & Type 1 & $\begin{array}{l}\text { Technology use: Average number of blood } \\
\text { glucose readings transferred was } 9.1 / \text { week. } \\
\text { Subjects who were identified as working in } \\
\text { a technical field sent more messages. } \\
\text { Clinical outcomes: Insulin dose of the } \\
\text { intervention group increased significantly. } \\
\text { Slight overall increase in HbA1c for both } \\
\text { groups (minor change in methodology of } \\
\text { HbA1c measurement occurred during the } \\
\text { study). }\end{array}$ \\
\hline $\begin{array}{l}\text { Wangberg } \\
\text { et al...8 } \\
2006\end{array}$ & $\begin{array}{l}\text { Parents of } \\
\text { children } \\
\text { with } \\
\text { diabetes }\end{array}$ & 11 & 11 weeks & $\begin{array}{l}\text { Randomized, test } \\
\text { the feasibility } \\
\text { and acceptability } \\
\text { of using SMS to } \\
\text { provide diabetes } \\
\text { information }\end{array}$ & Norway & $\begin{array}{l}\text { Not } \\
\text { reported }\end{array}$ & $\begin{array}{l}\text { SMS, } \\
\text { information/ } \\
\text { educational } \\
\text { messages }\end{array}$ & Type 1 & $\begin{array}{l}\text { Satisfaction/usefulness: Generally positive } \\
\text { feedback regarding the system. Findings } \\
\text { indicate the system was good at providing } \\
\text { new and updated information about } \\
\text { diabetes and information about the } \\
\text { parents' role in managing a child's diabetes } \\
\text { to child's self-management. }\end{array}$ \\
\hline $\begin{array}{l}\text { Hanauer } \\
\text { et al. }{ }^{29} \\
2009\end{array}$ & Patients & $\begin{array}{l}40 \text { ( } 22 \text { in text } \\
\text { message } \\
\text { group/18 in } \\
\text { e-mail group) }\end{array}$ & 12 weeks & $\begin{array}{l}\text { Randomized, } \\
\text { usability, } \\
\text { preference, blood } \\
\text { glucose levels }\end{array}$ & $\begin{array}{l}\text { Not } \\
\text { reported }\end{array}$ & $\begin{array}{l}\text { Subjects } \\
\text { used their } \\
\text { own }\end{array}$ & $\begin{array}{l}\text { Application, } \\
\text { SMS, } \\
\text { reminder, } \\
\text { diary }\end{array}$ & $\begin{array}{l}\text { Not } \\
\text { reported }\end{array}$ & $\begin{array}{l}\text { Technology use: } 18 \text { subjects used the text } \\
\text { messaging system; } 11 \text { of the e-mail } \\
\text { subjects used the system. Females were } \\
\text { more likely to use the system. Cell phone } \\
\text { group requested more reminders, } \\
\text { responded faster to reminders, and } \\
\text { submitted significantly more blood glucose } \\
\text { test results. Between-group difference } \\
\text { decreased as time went on. } \\
\text { Satisfaction/usefulness: Half of all subjects } \\
\text { reported they would prefer the cell phone; } \\
17 \% \text { preferred the e-mail; } 10 \% \text { wanted } \\
\text { both; two subjects did not prefer either. } \\
\text { Clinical outcomes: No difference in glycemic } \\
\text { control between groups was found. }\end{array}$ \\
\hline $\begin{array}{l}\text { Kollmann } \\
\text { et al.., } \\
2007\end{array}$ & Patients & 10 & 12 weeks & $\begin{array}{l}\text { Usage, } \\
\text { satisfaction, } \\
\text { HbA1c, blood } \\
\text { glucose level }\end{array}$ & $\begin{array}{l}\text { Not } \\
\text { reported }\end{array}$ & Yes & $\begin{array}{l}\text { Application, } \\
\text { SMS, } \\
\text { reminder, } \\
\text { diary }\end{array}$ & Type 1 & $\begin{array}{l}\text { Technology use: } 3,850 \text { log-ins were } \\
\text { registered over the entire program; } 13,003 \\
\text { datasets were transmitted; } 85 \% \text { adherence } \\
\text { rate of sending at least three blood glucose } \\
\text { values daily. An average log-on and data } \\
\text { transfer took approximately } 3 \text { min. } \\
\text { Satisfaction/usefulness: Application was } \\
\text { easy to learn and use. } \\
\text { Clinical outcomes: Significant decrease in } \\
\text { HbA1c; decrease in average blood glucose } \\
\text { level. }\end{array}$ \\
\hline $\begin{array}{l}\text { Rami } \\
\text { et al., } \\
2006\end{array}$ & Patients & 36 & 24 weeks & $\begin{array}{l}\text { Randomized, } \\
\text { crossover, } \\
\text { feasibility } \\
\text { and glycemic } \\
\text { control }\end{array}$ & $\begin{array}{l}\text { Not } \\
\text { reported }\end{array}$ & Yes & $\begin{array}{l}\text { Application, } \\
\text { SMS, } \\
\text { reminder, } \\
\text { diary }\end{array}$ & Type 1 & $\begin{array}{l}\text { Technology use: Nine patients sent only } \\
<50 \% \text { of the } 4 \text { daily messages. } \\
\text { Clinical outcomes: Group using phone } \\
\text { application demonstrated improved } \\
\text { glycemic control. } \\
\text { Satisfaction/usefulness: Some service area } \\
\text { coverage problems were reported by } 26 \\
\text { subjects. Patients reported overall } \\
\text { satisfaction with the application. Patients } \\
\text { did not like the additional workload or the } \\
\text { service area problems. }\end{array}$ \\
\hline
\end{tabular}




\section{HOLTZ AND LAUCKNER}

\begin{tabular}{|c|c|c|c|c|c|c|c|c|c|}
\hline $\begin{array}{l}\text { AUTHOR, } \\
\text { YEAR }\end{array}$ & $\begin{array}{l}\text { SUBJECT } \\
\text { TYPE }\end{array}$ & $\begin{array}{l}\text { NUMBER } \\
\text { OF } \\
\text { SUBJECTS }\end{array}$ & $\begin{array}{c}\text { DURATION } \\
\text { OF } \\
\text { STUDY }\end{array}$ & $\begin{array}{l}\text { STUDY } \\
\text { TYPE }\end{array}$ & $\begin{array}{c}\text { STUDY } \\
\text { LOCATION }\end{array}$ & $\begin{array}{c}\text { PHONE } \\
\text { PROVIDED }\end{array}$ & $\begin{array}{c}\text { TYPE OF } \\
\text { TECHNOLOGY } \\
\text { AND } \\
\text { FUNCTION(S) } \\
\text { USED }\end{array}$ & $\begin{array}{c}\text { TYPE } \\
\text { OF } \\
\text { DIABETES }\end{array}$ & $\begin{array}{l}\text { SUMMARY } \\
\text { OF RESULTS }\end{array}$ \\
\hline $\begin{array}{l}\text { Rossi } \\
\text { et al.,., } \\
2009\end{array}$ & Patients & $\begin{array}{l}50 \text { (DID1) } \\
41 \text { (DID2) }\end{array}$ & 12 weeks & $\begin{array}{l}2 \text { pilot studies } \\
\text { (DID1 and DID2), } \\
\text { feasibility and } \\
\text { acceptability, } \\
\text { glycemic } \\
\text { outcomes }\end{array}$ & Italy & $\begin{array}{l}\text { Not } \\
\text { reported }\end{array}$ & $\begin{array}{l}\text { Application, } \\
\text { SMS, } \\
\text { reminder, } \\
\text { diary, } \\
\text { information/ } \\
\text { education }\end{array}$ & Type 1 & $\begin{array}{l}\text { DID1: Technology use: Patients sent } \\
\text { average of } 10.4 \text { messages per day. } \\
\text { Satisfaction/usefulness: Baseline } \\
\text { perceptions of the system were } \\
\text { good. } \\
\text { Patients were interested in trying it and } \\
\text { thought it could be helpful. Post-study } \\
\text { survey concluded the system led } \\
\text { to good satisfaction and was useful, } \\
\text { easy to use, and easy to learn. CHO } \\
\text { counting tool was ranked the most } \\
\text { important function, followed by insulin } \\
\text { bolus calculator, food diary, physical } \\
\text { activity diary, and food exchange. } \\
\text { Communication with provider was } \\
\text { ranked as being effective. Technical } \\
\text { issues included the speed. } \\
\text { Clinical outcomes: No significant } \\
\text { differences were shown in HbA1c, } \\
\text { blood pressure, BMI, DTSQ-WHO, } \\
\text { and SF-36. } \\
\text { Self-care: Subjects reported better } \\
\text { eating behaviors and reported higher } \\
\text { knowledge of diabetes. } \\
\text { DID2: Clinical outcomes: Fasting blood } \\
\text { glucose and postprandial blood glucose } \\
\text { decreased significantly. HbA1c decreased } \\
\text { (not significant). Short-acting insulin use } \\
\text { decreased. Long-acting insulin use } \\
\text { increased. }\end{array}$ \\
\hline $\begin{array}{l}\text { Tasker } \\
\text { et al.,., } \\
2007\end{array}$ & Patients & $\begin{array}{l}37 \text { (19 mobile } \\
\text { phone/18 } \\
\text { computer apps) }\end{array}$ & 4 weeks & $\begin{array}{l}\text { Randomized, } \\
\text { ascertain the rate } \\
\text { of hypoglycemia, } \\
\text { technology } \\
\text { preference }\end{array}$ & $\begin{array}{l}\text { United } \\
\text { Kingdom }\end{array}$ & $\begin{array}{l}\text { Subjects } \\
\text { used their } \\
\text { own }\end{array}$ & $\begin{array}{l}\text { SMS, } \\
\text { reminders, } \\
\text { diary }\end{array}$ & Type 1 & $\begin{array}{l}\text { Technology use: } 58 \text { responses; } 132 \\
\text { hypoglycemic reports over } 705 \\
\text { recorded days. Response rate } \\
\text { of the mobile phone group was } \\
95 \% \text {, paper diary } 65 \% \text {, and computer } \\
\text { application } 89 \% \text {. } \\
\text { Satisfaction/usefulness: } \\
\text { High preference for using } \\
\text { the mobile phone. } \\
\text { Clinical outcomes: } 5.2 \\
\text { hypoglycemia episodes/month; } \\
\text { all were mild. }\end{array}$ \\
\hline $\begin{array}{l}\text { Turner } \\
\text { et al., }{ }^{19} \\
2009\end{array}$ & $\begin{array}{l}\text { Patients, } \\
\text { HCPs }\end{array}$ & $\begin{array}{l}23 \text { (patient } \\
\text { subjects) }\end{array}$ & 12 weeks & $\begin{array}{l}\text { Utilization } \\
\text { and perceptions }\end{array}$ & $\begin{array}{l}\text { United } \\
\text { Kingdom }\end{array}$ & Yes & $\begin{array}{l}\text { Application, } \\
\text { Bluetooth, } \\
\text { reminder, } \\
\text { diary }\end{array}$ & Type 2 & $\begin{array}{l}\text { Technology use: } 160 \text { blood glucose } \\
\text { readings were transmitted per patient. } \\
\text { Satisfaction/usefulness: Providers reported } \\
\text { they liked the ability to access up-to date } \\
\text { information, potential to support patient } \\
\text { self-management, and enhancing patient } \\
\text { self-management. Technical problems were } \\
\text { also reported and addressed during the } \\
\text { study. } \\
\text { Clinical outcomes: Decrease in HbA1c } \\
\text { (not significant). } \\
\text { Self-care: Patients reported feeling more } \\
\text { in control of their diabetes, increased } \\
\text { confidence in self-managing } \\
\text { their diabetes. }\end{array}$ \\
\hline
\end{tabular}


DIABETES MANAGEMENT VIA MOBILE PHONES

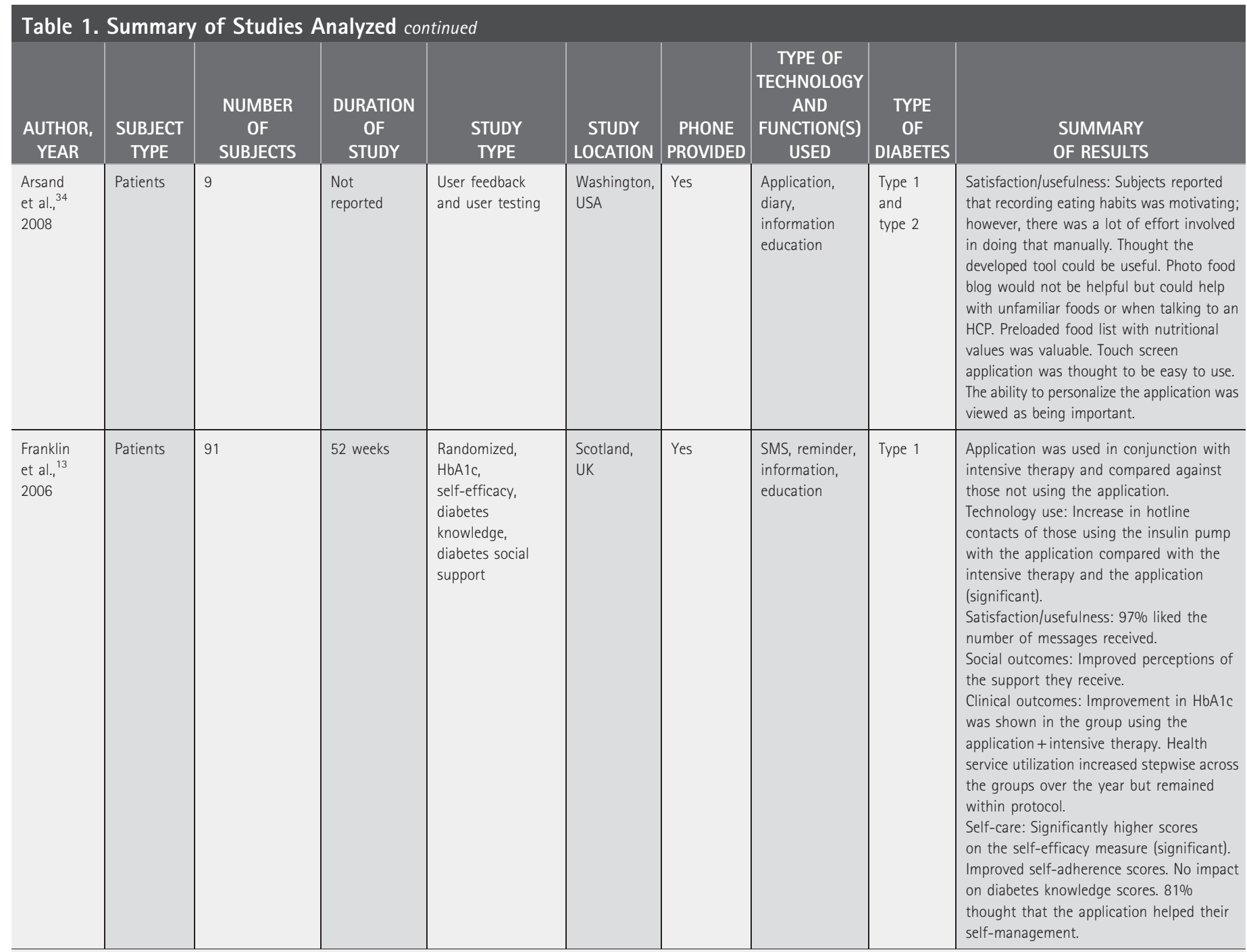

AMI, acute myocardial infarction; BMI, body mass index; CHO, carbohydrate; DTSO-WHO, Diabetes Treatment Satisfaction Questionnaire-World Health Organization; HbA1c, hemoglobin A1c; HCP, healthcare provider; RCT, randomized controlled trial; SF-36, 36-item Short Form; SMS, short message service.

limited. To move the field forward this issue should be addressed in all future studies. A limitation of this review, however, is that the assessment of outcomes reported was done at a more general level so as to allow for comparison across studies. HbA1c was commonly monitored, but other condition-specific factors assessed by the studies were not completely addressed in this review. It may be the case that the outcomes were more promising for other parameters not discussed here.

Short intervention periods also may have impacted the reported outcomes. For example, the two studies ${ }^{13,14}$ that had a study period of at least a year were able to demonstrate a significant effect of the intervention on key outcomes. Additionally, because of the relatively short time frames of most studies, we are unable to determine the long-term impacts of mobile phone diabetes interventions, including retention, adherence, sustainability, and integration into the healthcare system. As diabetes is a condition that requires lifelong management and monitoring, longer intervention lengths may provide better insights.

It is interesting that reports of provider interactions with the patients using the mobile phones were limited in these studies. It is not clear how additional data regarding a patient's diabetes were presented to either the patient's physician or nurse and how medical professionals then integrated this information into their practice. Patients' perceptions of usefulness were reported in just under half (48\%) of the studies, with most (90\%) reporting that the patients perceived the application positively. However, past research has demonstrated that gatekeepers to the introduction of new technologies are often the healthcare providers. ${ }^{15}$ Therefore, it is important to understand providers' perceptions of the challenges and barriers to integrating new technologies that might help improve patient 


\section{HOLTZ AND LAUCKNER}

\section{Table 2. Coding Definitions and Frequencies \\ ITEMS \\ DOMAIN \\ CODED \\ FREQUENCY N}

Self-care management

The care taken by

individuals toward their

own health and well-

being, the actions they

take to lead a healthy

lifestyle

Use of application

\begin{tabular}{l|l|l|l}
\hline $\begin{array}{l}\text { How the main } \\
\text { intervention was provided } \\
\text { to the participants }\end{array}$ & Application & $71.4 \%$ & 15 \\
\hline
\end{tabular}

Method of data transmittal

If an application was used, how data was

transferred to

the phone

\begin{tabular}{l|l|r|r} 
& Both & $4.8 \%$ & 1 \\
\hline Not used & Hand-entered & $38.1 \%$ & 8 \\
\hline
\end{tabular}

Intervention activity

\begin{tabular}{|c|c|c|c|}
\hline \multirow{4}{*}{$\begin{array}{l}\text { The type of activity that } \\
\text { the intervention provided } \\
\text { through use of the mobile } \\
\text { phone }\end{array}$} & Reminder & $52.4 \%$ & 11 \\
\hline & Diary & $81.0 \%$ & 17 \\
\hline & Entertainment & $4.8 \%$ & 1 \\
\hline & Information & $28.6 \%$ & 1 \\
\hline
\end{tabular}

Study design

\begin{tabular}{|c|c|c|c|}
\hline $\begin{array}{l}\text { Were patients } \\
\text { randomized to } \\
\text { a study condition? }\end{array}$ & Randomized & $47.6 \%$ & 10 \\
\hline \multicolumn{4}{|l|}{ Study duration } \\
\hline \multirow{2}{*}{$\begin{array}{l}\text { How long did } \\
\text { the study last? }\end{array}$} & Reported & $90.5 \%$ & 19 \\
\hline & In weeks, average & 22.5 & \\
\hline
\end{tabular}

Type of diabetes

Was the intervention developed for a specific type of diabetes?

Outcomes

What outcomes did the study examine? If they examined the outcome, what there a change?

\begin{tabular}{|l|c|c|}
\hline Type 1 & $57.1 \%$ & 12 \\
\hline Type 2 & $19.0 \%$ & 4 \\
\hline Both & $9.5 \%$ & 2 \\
\hline Not reported & $14.3 \%$ & 3 \\
\hline BMl reported & $23.8 \%$ & 5 \\
\hline Improvement & $80.0 \%$ & 4 \\
\hline No change & $19.0 \%$ & 1 \\
\hline Knowledge reported & $14.3 \%$ & 3 \\
\hline Improvement & $66.7 \%$ & 2 \\
\hline No change & $33.3 \%$ & 1 \\
\hline
\end{tabular}

Table 2. continued
\begin{tabular}{|c|c|c|c|}
\multicolumn{2}{|c|}{$\begin{array}{c}\text { ITEMS } \\
\text { CODED }\end{array}$} & FREQUENCY & $N$ \\
\cline { 2 - 4 } & Satisfaction reported & $47.6 \%$ & 10 \\
\cline { 2 - 4 } & Satisfactory/useful & $90.0 \%$ & 9 \\
\cline { 2 - 4 } & Mixed & $10.0 \%$ & 1 \\
\cline { 2 - 4 } & Self-efficacy reported & $23.8 \%$ & 5 \\
\cline { 2 - 4 } & Improvement & $100.0 \%$ & 5 \\
\cline { 2 - 4 } & No change & $0.0 \%$ & \\
\cline { 2 - 4 } & HbA1c reported & $61.9 \%$ & 13 \\
\cline { 2 - 4 } & Improvement & $84.6 \%$ & 11 \\
\cline { 2 - 4 } & No change & $7.7 \%$ & 1 \\
\cline { 2 - 4 } & Deterioration & $7.7 \%$ & 1 \\
\hline
\end{tabular}

Sample size

\begin{tabular}{|c|c|c|c|}
\hline \multirow{2}{*}{$\begin{array}{l}\text { Was the sample size } \\
\text { reported? What was the } \\
\text { sample size? }\end{array}$} & Reported & $100.0 \%$ & 21 \\
\hline & Average & 38 & \\
\hline Recruitment process & \multicolumn{3}{|l|}{ Inclusion criteria } \\
\hline \multirow{12}{*}{$\begin{array}{l}\text { Did the study report } \\
\text { specific inclusion and } \\
\text { exclusion criteria? } \\
\text { What were they? }\end{array}$} & Age & $61.9 \%$ & 13 \\
\hline & Severity of diabetes & $28.6 \%$ & 6 \\
\hline & Length of diagnoses & $23.8 \%$ & 5 \\
\hline & Insulin treatment & $33.3 \%$ & 7 \\
\hline & Access to Internet & $14.3 \%$ & 1 \\
\hline & Ability to use phone & $19.0 \%$ & 4 \\
\hline & \multicolumn{3}{|l|}{ Exclusion criteria } \\
\hline & Comorbidities & $23.8 \%$ & 5 \\
\hline & Pregnancy & $14.3 \%$ & 3 \\
\hline & Mental illness & $9.5 \%$ & 2 \\
\hline & $\begin{array}{l}\text { Not willing to monitor } \\
\text { diabetes }\end{array}$ & $4.8 \%$ & 1 \\
\hline & $\begin{array}{l}\text { Lived outside } \\
\text { of coverage area }\end{array}$ & $4.8 \%$ & 1 \\
\hline
\end{tabular}

Phone ownership

\begin{tabular}{l|l|c|c}
\hline Who provided the phone? & Study provided phone & $57.1 \%$ & 12 \\
\cline { 2 - 4 } & Subjects use own phone & $14.3 \%$ & 3 \\
\cline { 2 - 4 } & Not reported & $28.6 \%$ & 6 \\
\hline
\end{tabular}

Study location

Was the location of the study explicitly stated?

\begin{tabular}{|l|l|l|}
\hline Reported & $71.4 \%$ & 15 \\
\hline
\end{tabular}


Table 2. Coding Definitions and Frequencies continued ITEMS

DOMAIN CODED FREQUENCY

Technical issues

\begin{tabular}{|c|c|c|c|}
\hline $\begin{array}{l}\text { Were technical issues } \\
\text { reported? }\end{array}$ & Reported & $66.7 \%$ & 14 \\
\hline \multicolumn{4}{|l|}{ Study participant } \\
\hline \multirow{4}{*}{$\begin{array}{l}\text { What group were } \\
\text { the participants } \\
\text { in the intervention? }\end{array}$} & Diabetes patient & $95.2 \%$ & 20 \\
\hline & Physician & $14.3 \%$ & 3 \\
\hline & Nurses & $4.8 \%$ & 1 \\
\hline & Informal caregiver & $9.5 \%$ & 2 \\
\hline \multicolumn{4}{|l|}{ Cost } \\
\hline $\begin{array}{l}\text { Were any cost data } \\
\text { provided? }\end{array}$ & Cost reported & $19.0 \%$ & 4 \\
\hline
\end{tabular}

outcomes. There may be some hesitation by a healthcare provider or facility in adopting this type of technology, as questions of reimbursement, privacy, and liability may be issues. However, these issues may be addressed by the Food and Drug Administration, which is taking steps to formally approve these types of applications for use in healthcare.

Many healthcare providers and organizations are searching for cost-effective ways of providing high-quality healthcare to patients, and using mobile phones may prove to be one effective strategy. However, cost issues were only mentioned in four of the studies, ${ }^{25,30-32}$ and none mentioned reimbursement issues. Cost analysis greatly depends on the equipment used and the timing associated with the intervention, but it will never be truly accurate without measuring the costs when implemented in the "real-world." Another real-world issue regards the provision of the mobile phones to the patients. Many of these studies provided the mobile phones to the patients, so it is unclear if they had their own phone and if the studies' technologies would have worked on them. Moreover, it is unknown if participants felt burdened when having to carry a second, unfamiliar phone, which could also lead to different patterns of usage than if they had used their own device. These issues are important for future studies to address if there are widespread implementation plans.

Overall, subjects' use and engagement in the mobile phone technologies utilized in the studies remain unclear. Some studies reported the number of text messages received and sent as an outcome measure, but it is impossible to gauge the actual level of patient engagement by this metric alone. Some studies only had a fraction of the participants respond to additional surveys that measured such outcomes. Additionally, in the studies that assessed the quantity and frequency of the messages, most report that a few highly active users sent the majority of the messages. Further inquiry is therefore needed to examine the characteristics of these highly active individuals and to work toward determining methods of encouraging other users to become more active. More engaged patients might demonstrate higher self-efficacy in managing their illnesses on a daily basis through use of mobile phones. In the studies reviewed, only a quarter (24\%) measured self-efficacy, ${ }^{13,16-19}$ with all reporting improvements. These improvements may lead to behavior change and better outcomes over time, but this is unlikely if the subjects do not continue using the technology or are not engaged in the activity.

Future research on the use of mobile phones for improving access and quality of healthcare is seemingly endless, as many mobile applications for health are being developed, and mobile phones continue to become more prevalent. However, many of these potential applications may be outside of the purview of an individual's healthcare team or insurance company; thus access to or awareness of quality or formally approved technologies could be limited. Future studies should examine healthcare providers' acceptance and intention to work mobile phone applications into their practices as well as the impact that these applications might have on patient-provider interaction. Furthermore, research could explore if there is a difference in outcomes between an individual independently deciding to use an application versus having an application recommended by his or her healthcare provider. It is also important to determine the characteristics of patients who would actually use the mobile phones and consequently benefit the most from their use in order to define appropriate referral strategies. Again, future study designs and implementation will need to address the long-term sustainability and outcomes for these types of applications. These types of research inquiries will help to inform the efficient and effective use of mobile phones in managing chronic diseases.

This systematic review has examined studies that used mobile phones to help people manage their diabetes. However, many of the studies evaluated did not use rigorous study designs, and few statistically significant results in patient outcomes were found. Nevertheless, there is promise in that many of the outcomes had positive trends, such as for HbA1c levels, self-efficacy, and diabetes knowledge. Altogether, the studies analyzed suggest that, despite the promise of using mobile phones in this area, much more rigorous research needs to be completed if these technologies are to be proven useful in the management of diabetes.

\section{Acknowledgments}

The authors would like to thank Sarah Krein, Ph.D., for her insight and feedback.

\section{Disclosure Statement}

No competing financial interests exist.

\section{REFERENCES}

1. Centers for Disease Control and Prevention. National diabetes fact sheet: National estimates and general information on diabtes and prediabetes in the United States, 2011. Atlanta: Centers for Disease Control and Prevention, U.S. Department of Health and Human Services, 2011.

2. World Health Organization. Diabetes programme: Facts \& figures. 2010. www.who.int/diabetes/facts/world_figures/en/ (last accessed May 11, 2011). 


\section{HOLTZ AND LAUCKNER}

3. Jones $H$, Edwards $L$, Vallis $T$, et al. Changes in diabetes self-care behaviors make a difference in glycemic control: The Diabetes Stages of Change (DiSC) Study. Diabetes Care 2003;26:732-737.

4. Karter A, Ackerson L, Darbinian J, et al. Self-monitoring of blood glucose levels and glycemic control: The Northern California Kaiser Permanente Diabetes Registry. Am J Med 2001;111:1-9.

5. International Telecommunications Union. The world in 2010: ICT facts and figures. Geneva: International Telecommunications Union, 2010.

6. Lenhart $A$, Ling R, Campbell $S$, Purcell K. Teens, cell phones and texting: Text messaging explodes as teens embrace it as the centerpiece of their communication strategies with friends. Washington, DC: Pew Internet \& American Life Project, 2010.

7. Fox S. Mobile health 2010. Washington, DC: Pew Research Center's Internet \& American Life Project, 2010.

8. Fjeldose $B$, Marshall A, Miller Y. Behavior change interventions delivered by mobile phone telephone short-message service. Am J Prev Med 2009;36:162-173.

9. Holtz B, Whitten P. Managing asthma with mobile phones: A feasibility study. Telemed J E Health 2009;15:907-909.

10. Krishna S, Boren SA, Balas E. Healthcare via cell phones: A systematic review. Telemed J E Health 2009;15:231-240.

11. Lim M, Hocking J, Hellard M, Aitken C. SMS STI: A review of the uses of mobile phones text messaging in sexual health. Int J STD AIDS 2008;19:287-290.

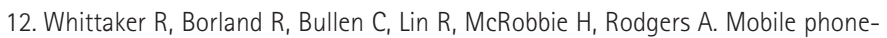
based interventions for smoking cessation. Cochrane Database Syst Rev 2009;(4):CD006611.

13. Franklin V, Waller A, Pagliari C, Greene S. A randomized controlled trial of Sweet Talk, a text-messaging system to support young people with diabetes. Diabet Med 2006;23:1332-1338.

14. Vähätalo $M$, Virtamo H, Viikari J, Ronnemaa T. Cellular phone transferred self blood glucose monitoring: Prerequisites for positive outcomes. Pract Diabetes Int 2004;21:192-194.

15. Whitten P, Holtz B. Provider utilization of telemedicine: The elephant in the room. Telemed J E Health 2008;14:995-997.

16. Faridi Z, Liberti L, Shuval K, Northrup V, Ali A, Katz D. Evaluating the impact of mobile telephone technology on type 2 diabetic patients' self-management: The NICHE pilot study. J Eval Clin Pract 2008;14:465-469.

17. Katz D, Nordwall B. Novel interactive cell-phone technology for health enhancement. J Diabetes Sci Techno/ 2008;2:147-153.

18. Quinn C, Sysko-Clough S, Minor J, Lender D, Okafor M, Gruber-Baldini A. WellDoc ${ }^{\mathrm{TM}}$ mobile diabetes management randomized controlled trial: Change in clinical and behavioral outcomes and physician satisfaction. Diabetes Technol Ther 2008;10:160-168.

19. Turner J, Larsen M, Tarassenko L, Neil A, Farmer A. Implementation of telehealth support for patients with type 2 diabetes using insulin treatment: An exploratory study. Inform Primary Care 2009;17:47-53.

20. Carroll A, Marrero D, Downs S. The HealthPia GlucoPack ${ }^{\mathrm{TM}}$ Diabetes Phone: A usability study. Diabetes Technol Ther 2007;9:158-164.

21. Istepanian R, Zitouni $K_{1}$, Harry $D$, et al. Evaluation of a mobile phone telemonitoring system for glycaemic control in patients with diabetes. J Telemed Telecare 2009;15:128-128.

22. Benhamou P, Melki V, Boizel R, et al. One-year efficacy and safety of web-based follow-up using cellular phone in type 1 diabetic patients under insulin pump therapy: The PumpNet Study. Diabetes Metab 2007;33:220-226.

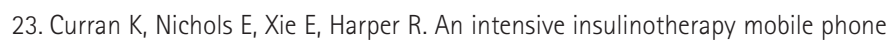
application built on artificial intelligence techniques. J Diabetes Sci Technol 2010;4:1-12.

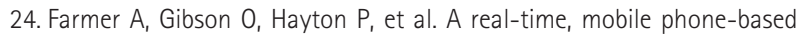
telemedicine system to support young adults with type 1 diabetes. Inform Primary Care 2005;13:171-177.

25. Ferrer-Roca 0, Cardenas A, Diaz-Carama A, Pulido P. Mobile phone text messaging in the management of diabetes. $J$ Telemed Telecare 2007;10:282286.

26. Franklin V, Greene G, Waller A, Pagliari C. Patients' engagement with "Sweet Talk"-a text messaging support system for young people with diabetes. $J$ Med Internet Res 2008;10:e20.

27. Gammon DA, Arsand E, Walseth O, Andersson N, Jenssen M, Taylor T. Parentchild interaction using a mobile and wireless system for blood glucose monitoring. J Med Internet Res 2005;7:e57.

28. Wangberg $S_{1}$ Arsand $E_{1}$ Andersson N. Diabetes education via mobile text messaging. J Telemed Telecare 2006;12(Suppl 1):55-56.

29. Hanauer D, Wentzell $K$, Laffel $N$, Laffel L. Computerized automated reminder diabetes system (CARDS): E-mail and SMS cell phone text messaging reminder to support diabetes management. Diabetes Technol Ther 2009;11:99-106.

30. Kollman A, Riedl M, Kastner P, Schreider G, Ludvik B. Feasibility of a mobile phone-based data service for functional insulin treatment of type 1 diabetes mellitus patients. J Med Internet Res 2007;9:e36.

31. Rami B, Popow C, Horn W, Waldhoer T, Schober E. Telemedical support to improve glycemic control in adolescents with type 1 diabetes mellitus. Eur J Pediatr 2006;165:701-705.

32. Rossi J, Nicolucci A, Pellegrini F, et al. Interactive diary for diabetes: A useful and easy-to-use new telemedicine system to support the decision-making process in type 1 diabetes. Diabetes Technol Ther 2009;11:19-24.

33. Tasker A, Gibson L, Franklin V, Gregor P, Greene S. What is the frequency of symptomatic mild hypoglycemia in type 1 diabetes in the young?: Assessment by novel mobile phone technology and computer based interviewing. Pediatr Diabetes 2007;8:15-20.

34. Arsand E, Tufano J, Ralston J, Hjortdahl P. Designing mobile dietary management support technologies for people with diabetes. $J$ Telemed Telecare 2008;14:329-332.
Address correspondence to: Bree Holtz, Ph.D. $H S R \& D$

Center for Clinical Management Research Veteran Affairs Ann Arbor Health Care System 2215 Fuller Road

Ann Arbor, MI 48105

E-mail: bree.holtz@va.gov

Received: June 15, 2011

Revised: July 25, 2011

Accepted: July 26, 2011 


\section{This article has been cited by:}

1. Omar El-Gayar, Prem Timsina, Nevine Nawar, Wael Eid. 2013. A systematic review of IT for diabetes self-management: Are we there yet?. International Journal of Medical Informatics . [CrossRef]

2. Linda Herbert, Victoria Owen, Lauren Pascarella, Randi Streisand. 2013. Text Message Interventions for Children and Adolescents with Type 1 Diabetes: A Systematic Review. Diabetes Technology \& Therapeutics 15:5, 362-370. [Abstract] [Full Text HTML] [Full Text PDF] [Full Text PDF with Links]

3. R. Nieuwlaat, J.-D. Schwalm, R. Khatib, S. Yusuf. 2013. Why are we failing to implement effective therapies in cardiovascular disease?. European Heart Journal 34:17, 1262-1269. [CrossRef]

4. Faraz S. Ahmad, Thomas Tsang. 2013. Diabetes Prevention, Health Information Technology, and Meaningful Use. American Journal of Preventive Medicine 44:4, S357-S363. [CrossRef]

5. Shantanu Nundy, Jonathan J. Dick, Marla C. Solomon, Monica E. Peek. 2013. Developing a behavioral model for mobile phonebased diabetes interventions. Patient Education and Counseling 90:1, 125-132. [CrossRef]

6. Josefien van Olmen, Grace Marie Ku, Maurits van Pelt, Jean Clovis Kalobu, Heang Hen, Christian Darras, Kristien Van Acker, Balthazar Villaraza, Francois Schellevis, Guy Kegels. 2013. The effectiveness of text messages support for diabetes self-management: protocol of the TEXT4DSM study in the democratic Republic of Congo, Cambodia and the Philippines. BMC Public Health 13:1, 423. [CrossRef] 